\title{
A MODIFIED THRING-NEWBY SCALING CRITERION FOR CONFINED, RAPIDLY SPREADING, AND UNSTEADY JETS
}

\author{
J. J. PARHAM* \\ G. J. NATHAN \\ S. J. HILL \\ P. J. MULLINGER
}

Department of Mechanical Engineering, Adelaide University, Adelaide, Australia

\begin{abstract}
Burner systems employing rapidly spreading jets have received considerable attention and found niche markets in combustion applications. However, there is limited information on their scalar mixing characteristics and none under confined conditions. Existing scaling criteria for the prediction of industrial flames using physical or computational models have limited application to practical combustion systems employing such burners. New scalar concentration measurements are obtained for confined precessing jet flows, a class of rapidly spreading jet, using planar laser-induced fluorescence. The measurements assess the effects of coflow and confinement to provide new physical insight and empirical data for the development of physical and computational models. The scalar measurements are used to derive a new scaling procedure for these flows. Compared with existing criteria, the proposed scaling method achieves improved mixing similarity between two systems employing different fluids at different scales. This is accomplished by distorting the
\end{abstract}

Received 9 February 2004; accepted 17 November 2004.

The assistance of Associate Professor Keith King and Dr. Zeyad Alwahabi in securing and configuring the Infinity Nd:YAG laser is most appreciated. Help and advice from Mr. Philip Cutler, Dr. David Nobes, and Dr. Barrie Jenkins was invaluable in setting up the experiments. This research was supported by the financial assistance of FCT-Combustion Pty. Ltd. and the Australian Research Council through the Collaborative Grants Scheme.

*Address correspondence to jordanp@fctinternational.com 
confinement ratio according to a modified form of the well-known Thring-Newby parameter and by incorporating an additional distortion to the stoichiometry. The approach is general and can also be applied to other rapidly spreading jet flows given appropriate empirical data.

Keywords: scaling, confined, unsteady, jet, scalar

\section{INTRODUCTION}

Most practical combustion systems employ turbulent diffusion flames whose spread and length are restricted by confining surfaces to which heat is transferred. In most cases, there is also a requirement that direct impingement of the flame onto these surfaces is avoided to prevent damage. Prediction of the flame envelope is therefore of direct interest in its own right as well as being a necessary component in the prediction of heat transfer characteristics and pollutant emissions.

A confined combustion application of particular interest, due to its relatively simple geometry, well-defined boundary conditions, and its significance as an industrial application, is the rotary kiln. A rotary kiln and burner system can be idealized as a jet of fuel issuing into a concentric confining cylinder with a surrounding uniform coflow of air, although the coflow in real rotary kilns is never truly uniform. These kilns are used in the processing of a wide range of materials that require high radiant heat transfer rates to drive the chemical reactions which transform the raw materials. Because of the high temperatures required in rotary kilns, they contribute significantly to global atmospheric pollution in the form of relatively high $\mathrm{NO}_{\mathrm{x}}$ emissions and the emission of dust and carbon dioxide from the raw feed in cement kilns (Ellis, 2000). In addition, the cost of energy is significant-for example, it can represent up to $50 \%$ of the cost of cement production. Hence, to satisfy the ongoing legislative and commercial pressures for decreased pollutant emission levels and increased energy efficiency, the optimization of combustion to match the requirements of a particular rotary kiln has become paramount. Consequently, the modeling of an axisymmetric confined jet configuration is of both fundamental and practical significance.

Because, to a first order, mixing controls the primary combustion processes in industrial-scale diffusion flames, considerable effort has also been devoted to the development of mixing devices that beneficially 
modify the mixing. Perhaps the three most relevant practical mixing devices in rotary kilns are high momentum, swirl, and precessing jet (PJ) nozzles (Nathan et al., 1998). Of these, the latter two have increased spreading rates relative to a simple jet. Bluff-body burners are rarely used in rotary kilns because of their poor reliability in such a high-temperature environment. PJ nozzles have found increased application of late because they typically provide a $40 \%$ reduction in $\mathrm{NO}_{x}$ emissions and a $2-5 \%$ reduction in specific fuel consumption over both swirl (Manias and Nathan, 1993, 1994) and high-momentum jet nozzles (Nathan and Manias, 1995; Videgar, 1997) in gas-fired rotary kilns due to their increased radiation. From a modeling perspective, they also offer some simplifications over swirl nozzles because they produce no net swirl and no near-field flow reversal. Nevertheless the near-field of a PJ flow is highly three-dimensional and unsteady, although only a small fraction of the combustion occurs in this region (Newbold, 1997).

Prediction of the scalar field in a flame (i.e., the concentrations of "fuel" and "air") is necessary for the prediction of both its heat release and emissions. Such predictions can be achieved by numerical simulations using computational fluid dynamics (CFD) or by scaling the measurements from small-scale experimental models. Despite the recent growth in computational modeling, CFD still has significant limitations in the modeling of large-scale reacting flows in which unsteady flow phenomena are important, that is, where the instantaneous flows can be considerably different from the mean. Clearly this is the case for the $\mathrm{PJ}$ flow, which is highly unsteady and three-dimensional. In contrast, small-scale physical models are effective at reproducing unsteady flow phenomena, although they have other limitations. Experience shows that the modeling of industrial-scale reacting systems using small-scale isothermal physical models is often still technically the most cost-effective method for predicting and optimizing the flame shape and burnout in rotary kilns and other systems (Jenkins, 1998; Mullinger, 1999). However, isothermal data is necessary for the development and validation of any modeling approach and also provides insight into the underlying mechanisms that control the mixing and reactions.

Various scaling criteria have been devised for isothermal physical modeling of the aerodynamics and, using the "mixed is burnt" approximation, of the reactions in enclosed industrial combustion systems. The most widely used criteria for scaling the mixing characteristics of ducted jet systems are those developed by Thring and Newby (1953), Craya 
and Curtet (1958), and Becker et al. (1963). All three criteria employ analytical solutions to the Navier-Stokes and continuity equations in the far field of a simple jet. These solutions require that the momentum ratio of the coflow relative to the jet in the model matches that of the reacting system. This is achieved by correcting for the variable densities that are present in the combusting environment by distorting the nozzle diameter relative to the duct diameter or the nozzle flows. While isothermal reduced-scale models may be locally correct, in terms of fuel/air mixing, they cannot replicate the effects of combustion on chemical reactions, volume changes, or buoyancy. However, Jenkins (1998) has shown that a simple correction to the isothermal data can be used to account for the effects of combustion and, hence, solve most industrial problems.

The analytical solutions employed in the Becker and Craya-Curtet parameters assume that the jet can be treated as a point source of momentum and that it spreads linearly from its source (i.e., that the entire flow is self-similar). Mi et al. (2001) have recently shown that, even for simple axisymmetric jets, near-field mixing rates are not the same as those in the far field and that both depend on the jet initial conditions. Furthermore, the virtual origin of the spreading rate is different from that of the decay rate, so that there can be no true virtual origin. Nevertheless, this assumption is reasonable for simple jet flows, and indeed the Becker and Craya-Curtet parameters have been validated for jet flows which can be treated as spreading linearly from a point source of momentum, such as a confined simple isothermal jet (Becker et al., 1963). For these jet flows, the Becker and Craya-Curter scaling criteria account for the effects of confinement better than the Thring-Newby parameter and require less distortion. Hence, Moles et al. (1972) recommend these criteria for modeling appropriate rotary kiln burner flames in which the jet flow can be treated as originating from a simple jet.

However, industrially relevant confined jet flows which seek to beneficially modify the mixing typically have a near-field spreading rate that is dramatically different from that in the far field. Under such conditions, the assumption that the jet spreads linearly from a point source of momentum ceases to be even a reasonable approximation. In these cases, Thring-Newby is the only applicable scaling criterion as it does not make any assumptions regarding the mixing conditions produced by a particular jet flow, as do the Craya-Curtet and Becker parameters. The Thring-Newby criterion maintains mixing similarity in the near field of the jet nozzle at the model and plant scale by keeping the momentum 
ratio of the coflowing fluid to jet fluid constant. New data is required to refine and improve the Thring-Newby scaling methodology for these industrially relevant rapidly spreading jets because no previous studies have assessed the effects of confinement and coflow on the scalar mixing field from rapidly spreading, practical jet flows.

In a similar vein, the development of new computational models also requires new data. The size and complexity of the mixing and reaction processes in a full-scale rotary kiln are such that direct numerical calculations will not be possible or cost-effective for many years. Hence, the modeling of $\mathrm{PJ}$ and other unsteady jet flows will inevitably require simplifying assumptions based on physical insight and the use of experimental data for calibration and/or validation (Nathan and Dally, 2003; Smith et al., 2003). Such data do not presently exist for rapidly spreading jets in general, and PJ flows in particular. For these reasons, the present study seeks to provide new scalar data and to develop a new experimentally derived scaling methodology that can be used in its own right and which will provide new insight into the dominant physical processes.

\section{PRECESSING JET FLOWS AND FLAMES}

The PJ nozzle is shown schematically in Figure 1. A fluid dynamic instability is generated naturally within a patented axisymmetric chamber following a large sudden expansion at its inlet (Luxton et al., 1991). When the dimensions of the chamber are within certain limits, the flow within the chamber reattaches asymmetrically, setting up an azimuthal pressure field which drives a precession of the reattaching jet within the chamber

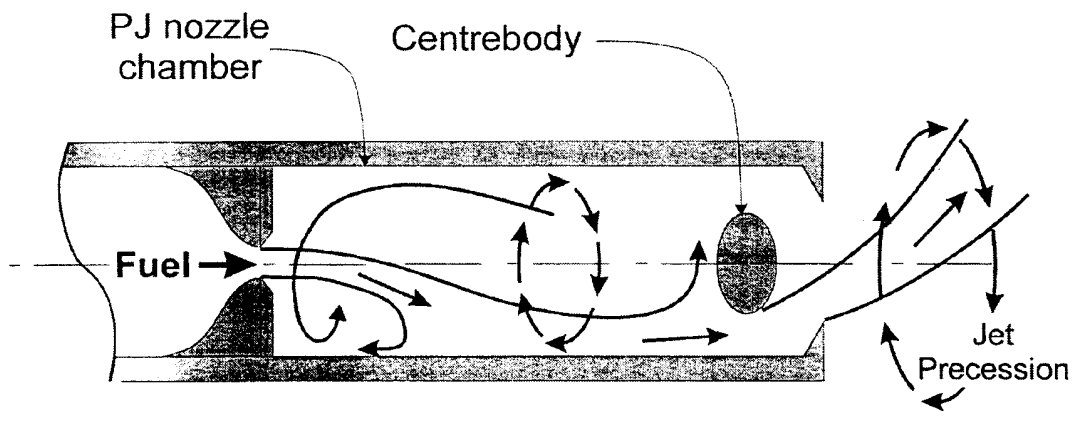

Figure 1. Schematic of the precessing jet nozzle. 
and the emerging jet (Nathan et al., 1998). Here "precession" refers the gyroscopic-like motion of the jet about an axis other than its own instantaneous axis. On leaving the chamber, the emerging jet is deflected across the nozzle axis at an angle of about $60^{\circ}$ by the pressure field in the exit plane and by interaction with the exit lip, and it is within the region immediately downstream from the nozzle exit that the flame is stabilized. The PJ nozzle design used in the present experiments also incorporates a centerbody that helps to stabilize the precessing flow model within the chamber (Wong et al., 2003).

The precession of the emerging jet has a dramatic influence on combustion. Nathan et al. (1996) assessed the effect of precession on combustion using a well-defined nozzle to mechanically rotate a round nozzle and so allow separation of the effects of precession from other possible influences of the fluidic device (Figure 1) on initial conditions. This showed that the type of flow depends strongly on the emerging Strouhal number, $S t_{\mathrm{p}}=\mathrm{f}_{\mathrm{p}} \times d_{\mathrm{e}} / u_{\mathrm{e}}$, where $f_{\mathrm{p}}$ is the frequency of precession, $d_{\mathrm{e}}$ is the exit diameter, and $u_{\mathrm{e}}$ is the exit velocity. However, for values of $S t_{\mathrm{p}}$ comparable with those of the fluidic device, the flame appeared similar and had the following properties: the lift of height was found to be reduced by a factor of 5 relative to the comparable nonprecessing jet, the flame width increased by a factor of 3 while the length was only slightly shorter, demonstrating an increase in flame volume, and flame radiation was increased slightly, consistent with visual appearance. In a subsequent study of the fluidic device, Newbold et al. (2000) compared the global mixing rates, radiant fraction, and emissions from a wide range of flames with different global mixing rates but identical fuel nozzle, fuel type, and fuel flow rate. The global mixing rate was determined from the flame volume and the throughput of fuel, whereas the emissions of $\mathrm{CO}, \mathrm{NO}_{\mathrm{x}}$, and radiation were measured following the work of Turns and Myhr (1991). The different burners included swirl jets, bluff-body jets, and a PJ, as well as a simple jet flame for reference. In all cases, consistent trends emerged. As the mixing rate was increased (causing a decrease in flame volume)-by the use of increased amounts of swirl or bluff-body air - the flame radiation decreased and thermal $\mathrm{NO}_{\mathrm{x}}$ increased. In contrast, the PJ was found to produce decreased global mixing rates (as demonstrated by increased flame volume), increased radiation, and reduced thermal $\mathrm{NO}_{\mathrm{x}}$. More recent measurements of soot volume fraction using laser-induced incandescence (Qamar et al., 2005) have confirmed these trends in soot formation by 
detailed measurements in three of the preceding flames. The amount of soot within the bluff-body flame was found to be an order of magnitude less than that of the simple jet flame, whereas that in precessing jet flame was a factor of about 3 higher. These trends in radiation and heat transfer have also been demonstrated at larger scale by Parham et al. (2000a), who performed a direct comparison of the flames from two burners in a pilot-scale rotary cement kiln simulator. They measured a total increase in radiant heat transfer, using a total radiation probe, by about $4 \%$ over an optimized high-momentum burner with some swirl.

\section{PROPOSED SCALING PROCEDURE}

Scaling uses experimental measurements to quantify "constants" of proportionality derived from dimensional arguments to allow results from one system to be related to those in another. Although complete similarity is never possible, useful predictions can be obtained from a small-scale model of a large combustion system using the "art of partial similarity" in which similarity of some dimensionless groups is relaxed, while that governing the dominant physical processes is maintained (Spalding, 1962).

Here the complex flow of a large-scale PJ nozzle is simulated using a small-scale nozzle and assuming Reynolds-number independence. Previous investigations by Nathan et al. (1998) have shown that the Strouhal number of geometrically similar nozzles is only weakly dependent upon Reynolds number, provided the nozzle inlet $R e>20,000$. Hence, the reduction in Reynolds number and physical scale are expected to have only a small influence on the Strouhal number of the emerging jet, as shown to be necessary by Nathan et al. (1996).

The scalar mixing of the PJ flow is characterized by measuring the mean centerline decay and jet spreading rate of concentration data, the latter characterized by the half-width, $r_{1 / 2}$, the radial location at which the concentration is half that at the centerline. The dominant dimensionless parameters effecting the flow are identified, allowing a new scaling relation to be devised to provide reasonable similarity of the mean concentration fields in isothermal and combusting environments. Note that the present model does not consider the effect of combustion itself on the decay rate or mixing rate of a flame as this is beyond the scope of the investigation. Furthermore, although the present data is specific, the procedure is general and can be applied to other practical mixing devices. 
The first stage in the scaling procedure is to maintain mixing similarity in the model and combustion facility by keeping the momentum ratio of the coflowing fluid to jet fluid constant. In an isothermal model of a combustion system this requires that the degree of confinement be distorted according to a slightly modified form of the calculation of Thring-Newby parameter, $\theta$ (Thring and Newby, 1953):

$$
\begin{gathered}
d_{\mathrm{or}}^{*}=\left(\frac{\rho_{0}}{\rho_{\mathrm{a}}}\right)^{0.5} d_{\mathrm{or}} \\
\theta=\frac{\dot{m}_{0}+\dot{m}_{\mathrm{a}}}{\dot{m}_{\mathrm{a}}} \cdot \frac{d_{\mathrm{or}}^{*}}{D_{\text {duct }}}
\end{gathered}
$$

Here the PJ nozzle inlet diameter, $d_{\text {or }}$, is chosen as the PJ reference length-scale; $D_{\text {duct }}$ is the kiln diameter at the plant scale or duct diameter at the model scale; $\rho_{\mathrm{a}}$ is the density of the coflowing air in the plant; $\rho_{0}$ is the density of the fuel at the PJ nozzle inlet (i.e., the fuel in a burner); and $m_{0}$ and $m_{\mathrm{a}}$ are the mass flow rates of the jet fluid and coflowing fluid, respectively. The distorted nozzle diameter, $d_{0}^{*}$, simulates a nozzle through which fluid of the density of the entrained fluid flows but with the same mass flow rate and momentum as that of the actual jet. In practice, the velocities and mass flow rates of the jet and coflow streams are substantially reduced in an isothermal model compared to the plant. Similarity of the Thring-Newby parameter is therefore satisfied according to Eq. (2) by keeping the product of the mass flux ratio and diameter ratio the same.

If the mass flux ratio is maintained constant between the full-scale and the model, the Thring-Newby criteria reduces to keeping the ratio between the distorted nozzle diameter and kiln diameter constant. However, when the Thring-Newby similarity criterion is used alone in an isothermal model of a reacting system with high preheated air temperatures, a significant increase in the nozzle to kiln diameter ratio occurs in the model. As mentioned earlier, the problem of such a distortion is more significant in rapidly spreading jets in a highly confined environment, because it would lead to increased impingement. Although other criteria have been developed to reduce the distortion, we seek an alternative approach because of the obvious advantages in seeking to preserve the mixing characteristics generated by the practical, rapidly spreading jet flows. This approach introduces an additional (correcting) distortion 
which is applied to the stoichiometric mixture fraction. The magnitude of this distortion is obtained empirically, and so requires new experimental data.

\section{EXPERIMENTAL TECHNIQUES AND PROCEDURES}

A systematic study of the effects of coflow velocity and confinement on the scalar mixing characteristics of a PJ flow was undertaken using planar laser-induced fluorescence (PLIF). PLIF can provide quantitative, spatially, and temporally resolved scalar data. As such, it is well suited to the highly transient and three-dimensional flow generated by the PJ nozzle.

The nozzle dimensions, flow rates, coflow velocities, and duct diameters used in the present experiments were selected to overlap with the scaled conditions from a number of combustion facilities. The primary reference for calculating the geometry and experimental conditions of the present experiments is the pilot-scale combustion facility used by Parham et al. (2000a), although the range of confinement and velocity ratios is large enough to span most of the anticipated conditions in rotary kilns (Parham, 2000).

A detailed description of the experimental technique is given by Parham (2000) and Parham et al. (2001). Briefly, the PJ nozzle was aligned on the axis of symmetry of a $0.5 \times 0.5 \mathrm{~m}$ cross section, closedcircuit water-tunnel (see Figure 2). Two PJ nozzles conforming to the optimal geometric requirements (Hill et al., 1992) were used in the present experiments. The nozzle used for the majority of the experiment had an upstream orifice diameter of $d_{\mathrm{or}}=7.5 \mathrm{~mm}$ and a chamber diameter of $d_{\mathrm{PJ}}=38 \mathrm{~mm}$. The second nozzle had an upstream orifice diameter of $d_{\mathrm{or}}=5.5 \mathrm{~mm}$ and a chamber diameter of $d_{\mathrm{PJ}}=28 \mathrm{~mm}$ and was nondimensionally identical to the $38-\mathrm{mm}$ PJ nozzle geometry. The Reynolds number at the upstream orifice of the PJ nozzles was constant for all experiments at $R e=66,200$ for the $38-\mathrm{mm} \mathrm{PJ}$ and $R e=62,400$ for the $28-\mathrm{mm}$ PJ. The jets were confined by acrylic ducts of internal diameters, $D_{\text {duct }}=290,390$, and $490 \mathrm{~mm}$, and $5-\mathrm{mm}$ wall thickness, which were mounted concentric with the nozzle centerline. The coflow velocity was controlled via the frequency of a recirculating pump.

The laser-dye system used by Karasso and Mungal $(1996,1997)$ was selected for the present PLIF experiments. The jet fluid was marked with $5(\& 6)$ carboxy- $2^{\prime}, 7^{\prime}$-dichlorofluorescein dye at a concentration of 


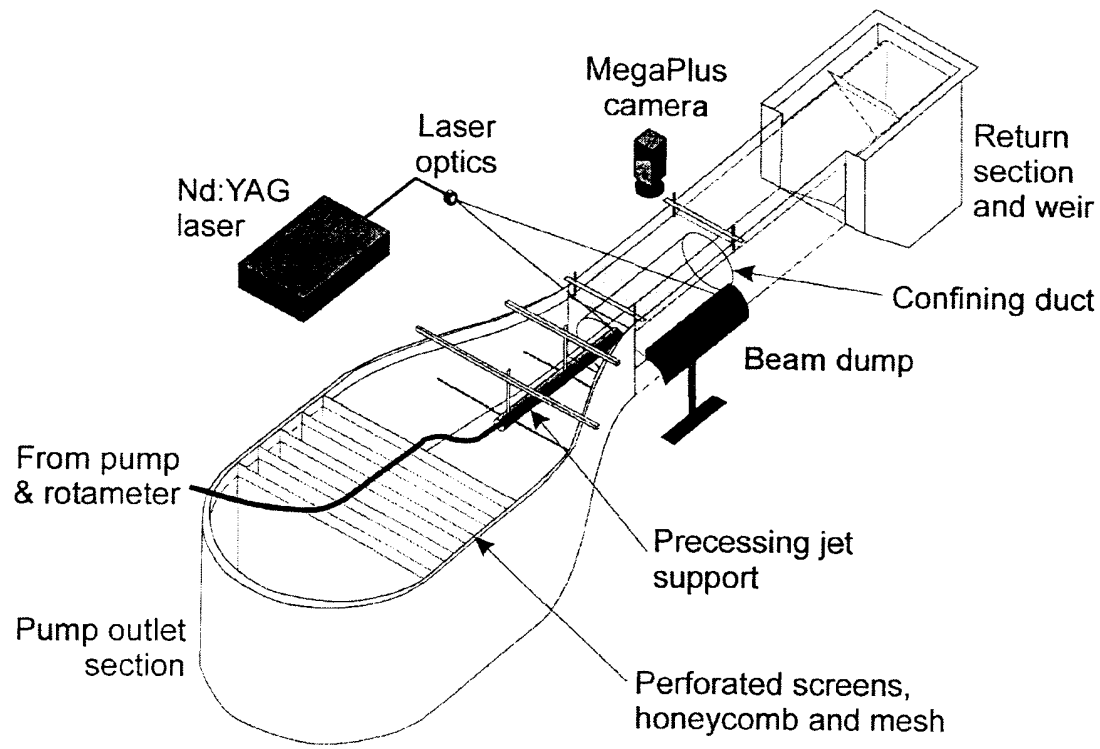

Figure 2. Schematic diagram of the experimental apparatus to investigate the effect of coflow and confinement on the mixing characteristics of the PJ nozzle by quantitative PLIF.

$7.9 \times 10^{-6} \mathrm{M}$. Excitation was provided by a horizontal laser sheet from a Coherent Infinity 40-100 Nd:YAG laser that was aligned with the jet centerline. The laser was operated at a power output of $250 \mathrm{~mJ} /$ pulse at $532 \mathrm{~nm}$ and a repetition rate of $6 \mathrm{~Hz}$.

A Kodak MegaPlus ES1.0 camera with a Fujinon CF12.5A lens captured the raw PLIF images. Scattered laser light was blocked by a longpass filter. Typically one to four batches of 152 images were collected for each condition. Suitable digital image processing was performed on each image to obtain a quantitative measurement of the jet fluid concentration, $\xi$. The spatial resolution is about $0.5 \times 0.5 \times 0.5 \mathrm{~mm}$, approximately five times larger than the Kolmogorov scale and eight times larger than $\lambda_{\mu}$, an "inner viscous scale" judged to be the most relevant length-scale for scalar data (Miller and Dimotakis, 1991), at the end of the measurement region. This implies that the scalar fluctuations will be somewhat underresolved but the mean data, which are all that we require here, will be reliable (Mi and Nathan, 2003). Furthermore, this resolution is comparable with that in numerous other PLIF investigations (Dahm and Dimotakis, 1990). 


\section{EXPERIMENTAL VALIDATION}

The linearity of the Nd:YAG/dichlorofluorscein PLIF technique was validated using a jet from a pipe with a length of more than 100 diameters ( $1000 \mathrm{~mm}$ long, $9.45 \mathrm{~mm}$ internal diameter brass pipe). An extensive analysis of the validation is presented by Parham (2000). The exit of the pipe was sharpened to a knife edge to render insignificant any possible external boundary-layer and bluff-body effects on the downstream mixing from the jet. The pipe was supported by two vertical airfoil sections mounted on the water tunnel by an overhead frame and aligned with the axis of symmetry of the working section. The cross section of the working section was $500 \times 500 \mathrm{~mm}$, so that the effects of confinement were negligible in the measurement region. The water tunnel was operated at a slight coflow, approximately $2 \%$ of the bulk jet exit velocity, and the Reynolds number at the jet exit based on the bulk jet velocity was $R e=28,200$.

The mean scalar characteristics of the validation pipe jet are compared with previous pipe jet measurements available in the literature in water $(S c \sim 1,000)$ and gases $(S c \sim 1)$ in Figure 3 . Figure $3 a$ shows the inverse scalar concentration on the jet centerline $1 / \bar{\xi}_{c}$, with the axial distance from the jet exit normalized to the momentum diameter, $d_{\varepsilon}$, which is given by the expression

$$
d_{\varepsilon}=\frac{2 M_{\mathrm{e}}}{\sqrt{\pi \rho_{\infty} J_{\mathrm{e}}}}
$$

where $M_{\mathrm{e}}=\int_{0}^{d / 2} 2 \pi \rho_{\mathrm{e}} U_{\mathrm{e}} r d r$ is the exit mass flux, $J_{\mathrm{e}}=\int_{0}^{d / 2} 2 \pi \rho_{\mathrm{e}} U_{\mathrm{e}}^{2} r d r$ is the exit momentum flux for the jet, $\rho_{\mathrm{e}}$ is the jet exit density, $\rho_{\infty}$ is the density of the surrounding fluid, and $U_{\mathrm{e}}$ is the mean jet exit axial velocity.

The present results demonstrate the expected relationship of inverse proportionality between the normalized distance from the nozzle, $\chi$, and the mean concentration, $\bar{\xi}_{\mathrm{c}}$. The relationship between the inverse mean concentration and normalized axial distance can be expressed as a linear equation, with the reciprocal of the slope corresponding to a concentration decay constant, $K_{1}$, and the intercept on the $x$ axis given by a virtual origin location, $x_{0,1} / d_{\varepsilon}$ (Mi et al., 2001). The value of the centerline concentration decay constant determined from these measurements, $K_{1}=6.36( \pm 5 \%)$, is higher than the majority of values reported in the literature, but is within the range of previous measurements. For example, it is very close to the value of $K_{1}=6.34$ measured by Papanicolou and List (1988) using a PLIF technique in water. The difference relative to other measurements is therefore 


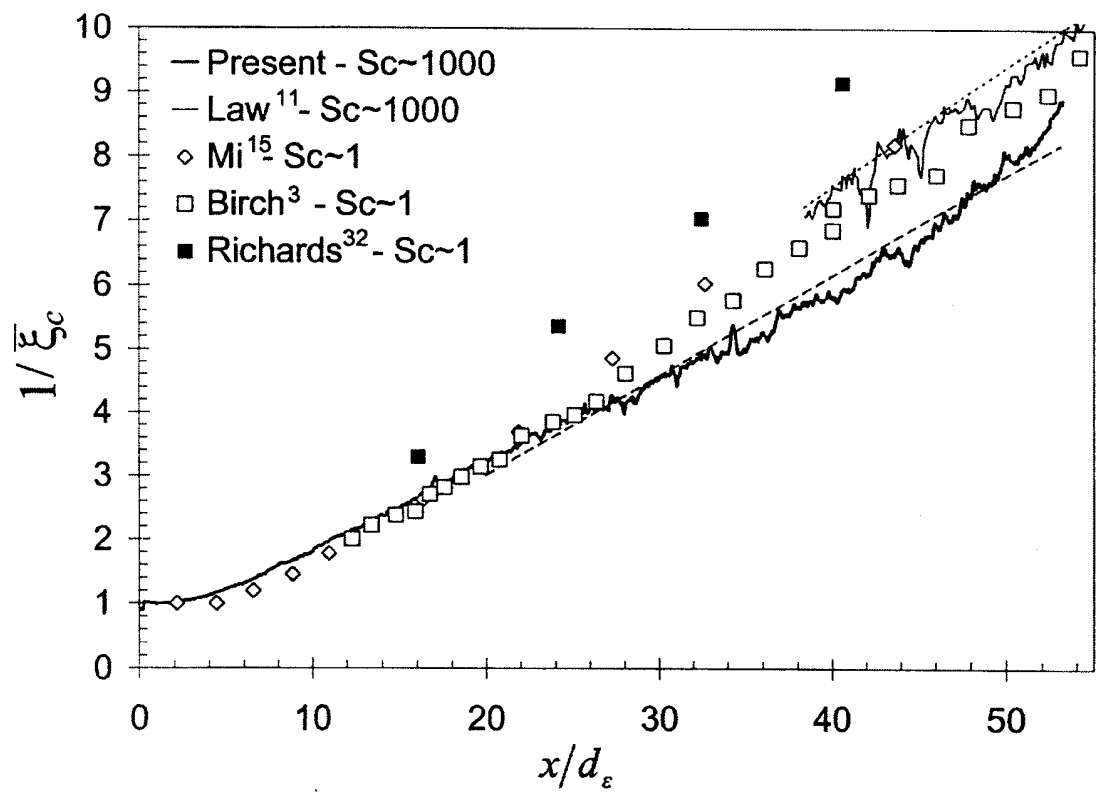

(a)

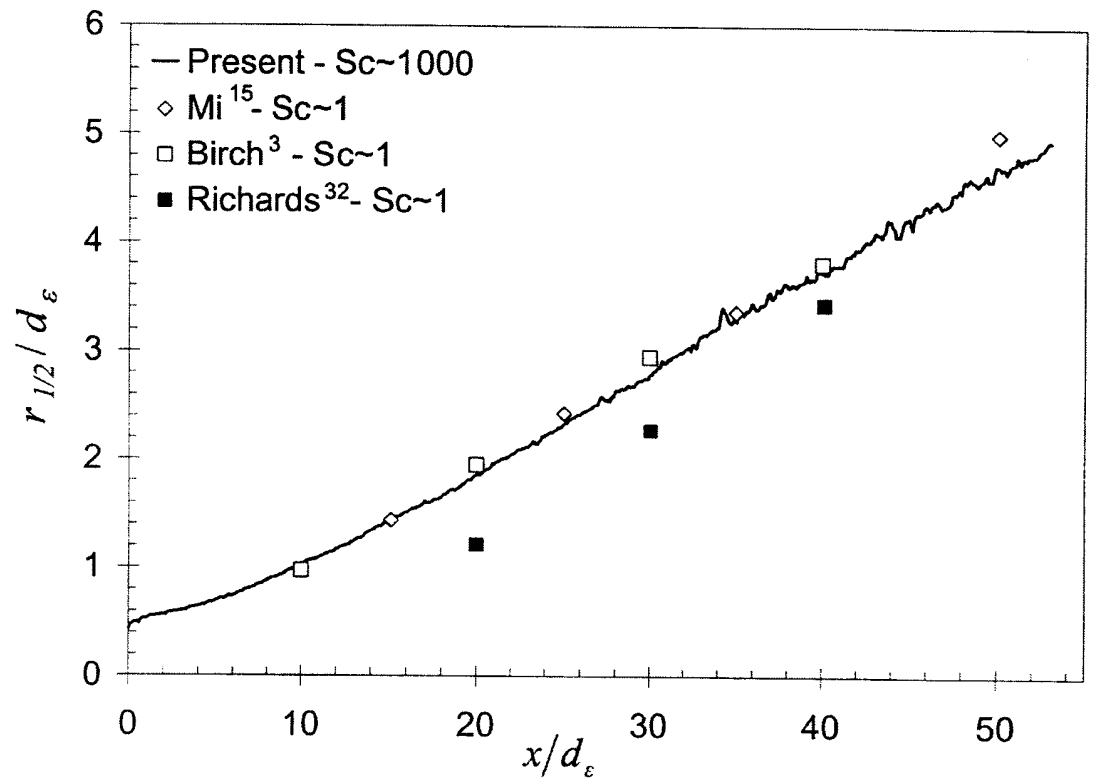

(b) 
attributed to differences in initial and boundary conditions such as the effects of the coflow velocity. The location of the virtual origin, $x_{0,1} / d_{\varepsilon}=0.84 \pm 1.9$, lies within the values reported in the literature of -0.35 to 4.73 for gaseous pipe jets.

The jet spread is quantified by determining the concentration halfwidth, $r_{1 / 2}$. The normalized half-width, $r_{1 / 2} / d_{\varepsilon}$, is plotted as a function of axial distance in Figure $3 b$ and compared with other data for pipe jets. This profile can similarly be described by a linear equation, with the slope given by the jet spreading rate, $K_{2}$, and the intercept on the $x$ axis given by a virtual origin location, $x_{0,2} / d_{\varepsilon}$ (Mi et al., 2001). The graph shows a constant rate of jet growth, $K_{2}=0.094( \pm 1 \%)$, which is at the lower end of the rates determined previously in gaseous and aqueous pipe jet experiments. This is consistent with the low rate of concentration decay along the jet centerline. The location of the virtual origin, $x_{0,2} / d_{\varepsilon}=0.05 \pm 0.4$, is within the range of values reported in the literature of -0.35 to 4.3 for gasphase pipe jet experiments.

The statistical analysis of the mean pipe jet scalar concentration just presented demonstrates that the results of the validation jet experiments are internally consistent and within the range of values obtained from previous measurements by other researchers. Differences are consistent with the wide range of different initial and boundary conditions between different investigations. Hence, the present implementation of the Nd:YAG/carboxydichlorofluorescein PLIF system is appropriate for the measurement of the passive scalar concentration from a jet.

\section{RESULTS}

High-quality instantaneous and mean images of the concentration field from a PJ nozzle have been published elsewhere (Parham, 2000; Parham et al., 2000b). Here, as examples we include a gray-scale instantaneous image and a mean image at the same conditions of jet velocity, coflow and confinement (see Figure 4). These images show that the instantaneous flow is very different from the mean flow field and is dominated

Figure 3. Results of the validation pipe jet experiment compared with the results of other liquid $(S c \sim 1000)$ and gaseous $(S c \sim 1)$ phase pipe jet investigations: $(a)$ inverse of the mean concentration on the jet centerline, $\vec{\xi}_{c}$, and $(b)$ the concentration half-width, $r_{1 / 2} / d$. Conditions: Reynolds number $=28,200$, coflow velocity $U_{\mathrm{a}}=0.06 \mathrm{~m} / \mathrm{s}$, jet diameter $=9.45 \mathrm{~mm}$, water-tunnel cross-section $=500 \times 500 \mathrm{~mm}$. 


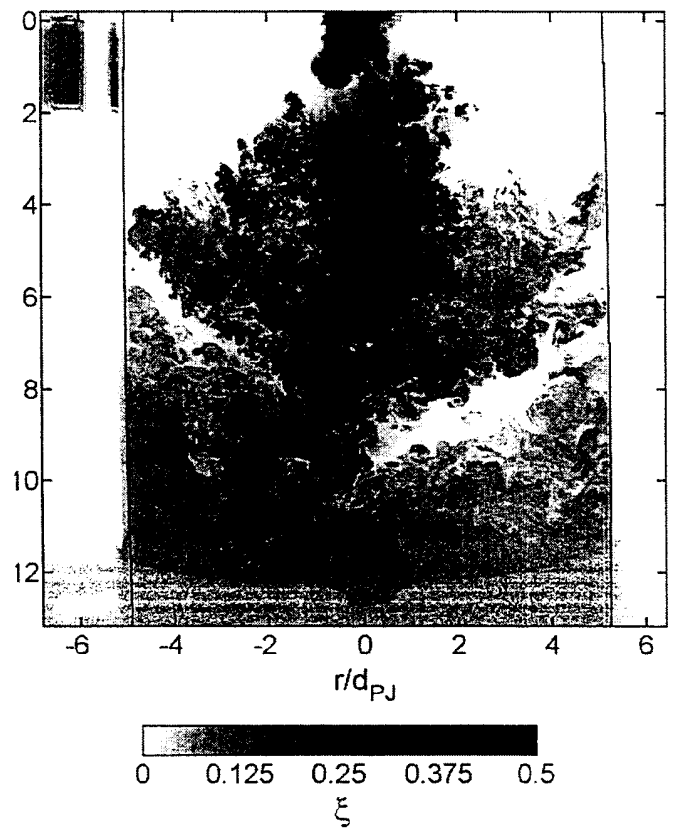

(a)
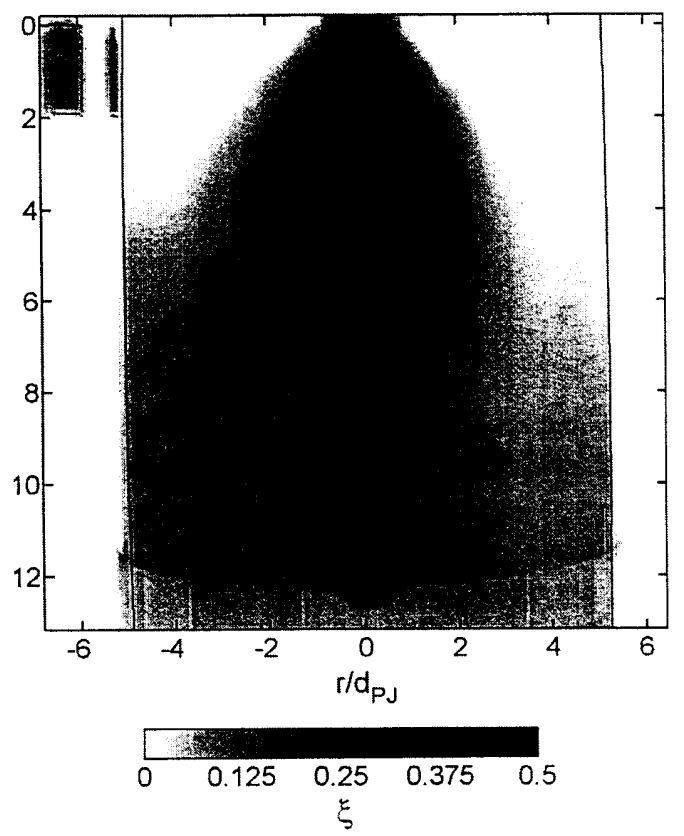

(b) 
by large-scale unsteady motions. The instantaneous jet emerges at a large angle to the nozzle axis and precesses, as described previously. The combined effect of these motions is to cause ambient fluid, shown as large light-colored pockets, to be entrained into the core of the jet very close to the nozzle exit. A complex interaction with the confining wall is also apparent. These features are difficult to reproduce computationally, especially with large Reynolds numbers and within the constraints of industrial budgets. They therefore highlight the relevance of physical modeling for such complex and unsteady flows.

Figure 5 shows that the mean concentration field is characterized by a transition from a near-field of rapid spread and decay to a second "developed" region of relatively linear decay. Here we adopt the term "developed" as distinct from "fully developed" to describe the second linear region within the present measurement range, since the mean characteristics display reasonable similarity while the higher order statistics do not. Furthermore, the effects of confinement, where present, necessarily prohibit self-similarity from being obtained and the "far field" is controlled by the duct, rather than the jet, and is beyond the region of investigation. Impingement of the jet on the confining walls is characterized by jet fluid moving slightly upstream and recirculating at the point of impingement, causing a local increase in the jet half-width (Figure $5 b$ ).

The transition between the near-nozzle and developed regions is characterized by an "elbow" in the centerline decay profile which occurs at approximately $x / d_{\mathrm{PJ}}=1.4$ (Figure $5 a$ ). After a very short "potential core," the rate of rapid decay in the near field is approximately constant and some four times that of a simple jet (Becker et al., 1967; Parham, 2000). Downstream from the "elbow," another region of approximately constant decay rate is observed which is approximately twice that of a simple jet. Although the concentration decay rate in these two regions is approximately linear, Figure $5 b$ shows that the change in slope of the half-width is gradual in the region $x / d_{\mathrm{PJ}}<6$, beyond which it is approximately constant. The mean radial concentration profiles are approximately Gaussian prior to the jet impinging on the duct walls

Figure 4. Grayscale images of the spatial distribution of $(a)$ instantaneous and $(b)$ mean jet concentration, $\xi$, from the $d_{\mathrm{PJ}}=38 \mathrm{~mm}$ PJ nozzle. Conditions: Reynolds number $=66,100$, $D_{\text {duct }} / d_{\mathrm{PJ}}=10.3, U_{\mathrm{a}} / U_{\mathrm{c}-\mathrm{PJ}}=0.035$. The band at the bottom of the images is the tape joining the two halves of the duct together. 

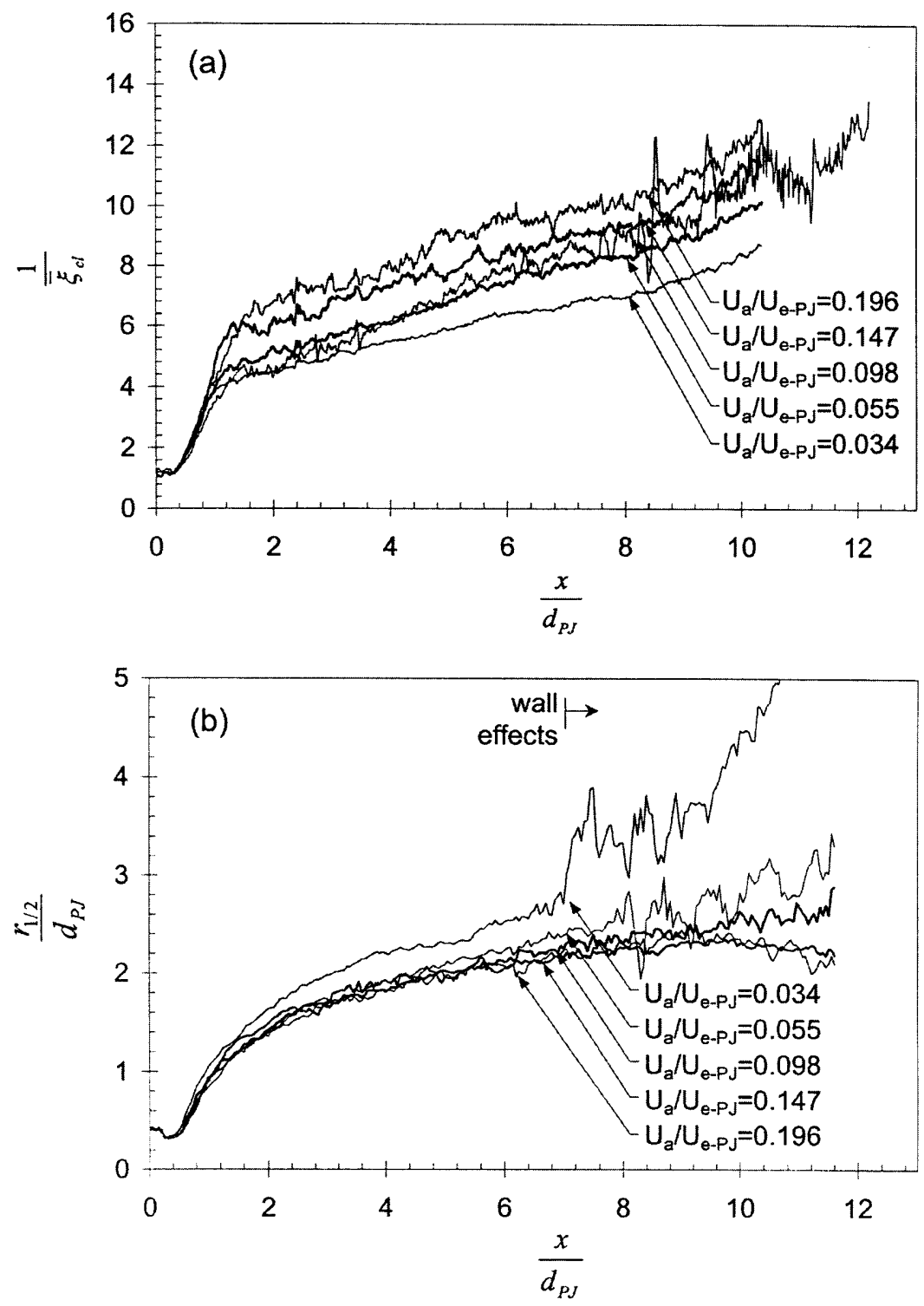

Figure 5. The effect of coflow velocity ratio on (a) the inverse mean scalar concentration on the jet centreline and $(b)$ the jet concentration half-width for a confinement of $D_{\mathrm{duct}} / d_{\mathrm{PJ}}=10.3$. Axial distance is normalized to the $\mathrm{PJ}$ nozzle chamber diameter. 
and show reasonable self-similarity for $x / d_{\mathrm{PJ}}>4$ (Parham, 2000), providing further justification for the use of the term "developed" to describe this region.

Figure $5 a$ shows that the rate of inverse concentration decay in the "developed" region is only weakly dependent on the coflow, although the dependence appears to be greater at higher coflow velocity ratios. However, the most obvious trend is that increasing the coflow acts to translate the inverse decay curve in the developed region to a higher value. Figure $5 b$ indicates that reducing the velocity ratio reduces the spread and the amount of impingement on the wall.

The effects of confinement on the mean concentration data from the PJ nozzle are shown in Figure 6. For a constant jet diameter, increasing the degree of confinement results in increased interaction with the walls and increased centerline decay rate in the developed region. However, if the duct diameter is used as the normalizing length scale instead of the nozzle diameter (Figure $6 a$ ), the concentration profiles collapse. This demonstrates that, downstream from the elbow point, the mean rate of mixing is controlled more strongly by the diameter of the duct rather than that of the jet. The data presented in Figure $6 a$, for $U_{\mathrm{a}} / U_{\mathrm{e}-\mathrm{PJ}}=0.035$, which is typical of other velocity ratios, also suggest that the centerline concentration decay rate, $K_{1}$, varies with the confinement ratio.

Figure $6 b$ similarly demonstrates that there are two alternative means of normalizing the effect of confinement on the jet concentration half-width. For the near-field, $x / d_{\mathrm{PJ}}<4$, the half-width profiles scale best with the PJ nozzle chamber diameter, whereas further downstream the profiles scale best with the duct diameter. Although it cannot be shown from Figure $6 b$ alone, the effect of confinement is reduced as the ratio of coflow velocity to jet velocity is increased and the concentration half-width contours collapse onto a single curve (Parham, 2000).

\section{Assessment of Dominant Dimensionless Parameters}

Given that the flow in the "developed" region displays reasonable similarity in the mean scalar data, we have selected it to characterize the PJ flow and assess the influence of confinement and coflow. This choice allows the use of the well-known free jet equations for the centerline concentration and jet half-width. Their simplicity is deemed to be 

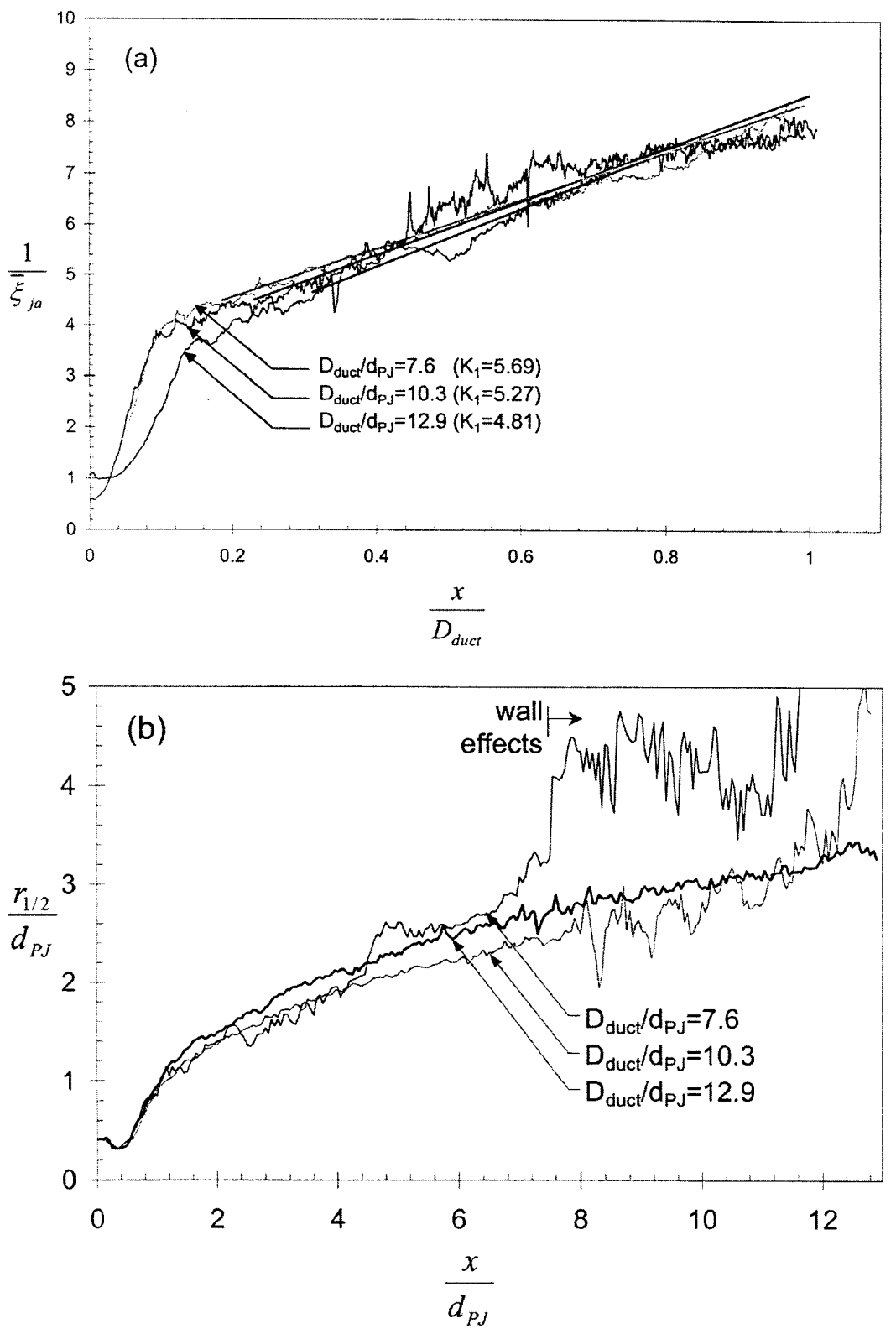

Figure 6. Typical examples of the effect of confinement on $(a)$ the inverse mean scalar concentration on the jet centreline, axial distance normalized to the diameter of the confining duct for a coflow velocity ratio of $U_{\mathrm{a}} / U_{\mathrm{e}-\mathrm{PJ}}=0.035$, and $(b)$ the jet concentration halfwidth for a coflow velocity ratio of $U_{\mathrm{a}} / U_{\mathrm{e}-\mathrm{PJ}}=\mathbf{0 . 0 5 5}$. 
appropriate for isolating the dominant dimensionless parameters required for developing a scaling methodology for the PJ nozzle.

The equation used to describe the mean concentration of jet fluid on the nozzle centerline of the developed region is

$$
\frac{1}{\xi_{\mathrm{c}}}=\frac{1}{K_{1}}\left(\frac{x-x_{0,1}}{l_{1}}\right)
$$

where $K_{1}$ is the decay constant, $x_{0,1}$ is the virtual origin, and $l_{1}$ is an appropriate length scale for normalizing the axial distance. The jet spread in the developed region can likewise be expressed as

$$
\frac{r_{1 / 2}}{l_{2}}=K_{2}\left(\frac{x-x_{0,2}}{l_{2}}\right)
$$

where $K_{2}$ is the spreading rate, $x_{0,2}$ is the jet-spread virtual origin, and $l_{2}$ is an appropriate length scale for normalizing the axial distance.

It was noted earlier that the dominant effect of increasing the coflow velocity ratio on the centerline decay of the developed region is to translate the curve upward. This corresponds to a shift in the virtual origin, $x_{0,1}$. It was likewise noted that the dominant effect of confinement on the centerline decay in the developed region is to modify the decay rate, $K_{1}$. Further analysis by Parham (2000) indicates that the most appropriate normalization length scale, $l_{1}$, is the duct diameter. The effectiveness of these major dependencies in characterizing the combined influences is assessed by plotting $K_{1}$ against the confinement ratio and $x_{0,1}$ against the coflow velocity ratio in Figure 7.

Figure $7 a$ confirms that $K_{1}$ depends approximately linearly on $D_{\text {duct }} / d_{\mathrm{PJ}}$. However, there is also a secondary, inconsistent dependence of $K_{1}$ on $U_{\mathrm{a}} / U_{\mathrm{e}-\mathrm{PJ}}$. Figure $7 b$ likewise confirms the strong dependence of $x_{0,1}$ on $U_{\mathrm{a}} / U_{\mathrm{e}-\mathrm{PJ}}$, along with a secondary dependence on $D_{\text {duct }} / d_{\mathrm{PJ}}$. Given that there is no simple method to account for the complex interdependence of these parameters, and in line with the goal of isolating the dominant dimensionless parameters, we accept the limitations of a simple, noninterdependent linear prediction. The linear prediction was based on a line of best fit plotted through all the data points shown on Figures 7 and 8 . The $R$-squared value, a measure of the scatter of data from the line of best fit, was used to quantify how well each line of best fit tracked the data and to determine the most appropriate scaling parameter. This corresponded to the linear prediction with the lowest 

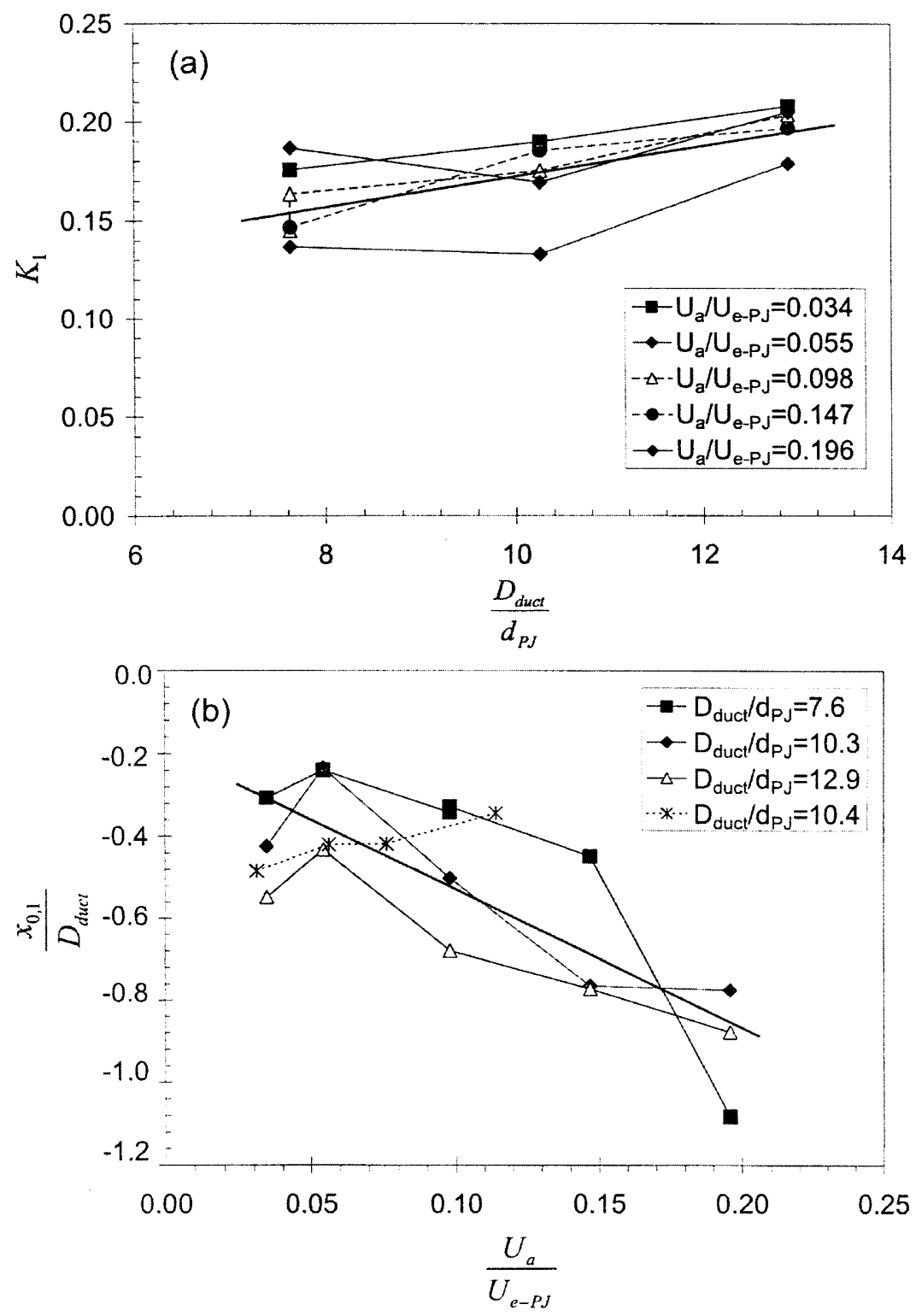

Figure 7. The effect of ( $a$ ) confinement on the concentration decay constant, $K_{1}$, for different coflow velocity ratios and constant $P J$ nozzle conditions and $(b)$ coflow velocity ratio on the far-field scalar concentration virtual origin, $x_{0,1} / D_{\text {duct }}$, for different confinement and constant PJ nozzle conditions. Solid lines indicate $d_{\mathrm{PJ}}=38 \mathrm{~mm}$ PJ nozzle results; dashed lines indicate $d_{\mathrm{PJ}}=28 \mathrm{~mm} \mathrm{PJ}$ nozzle results. 

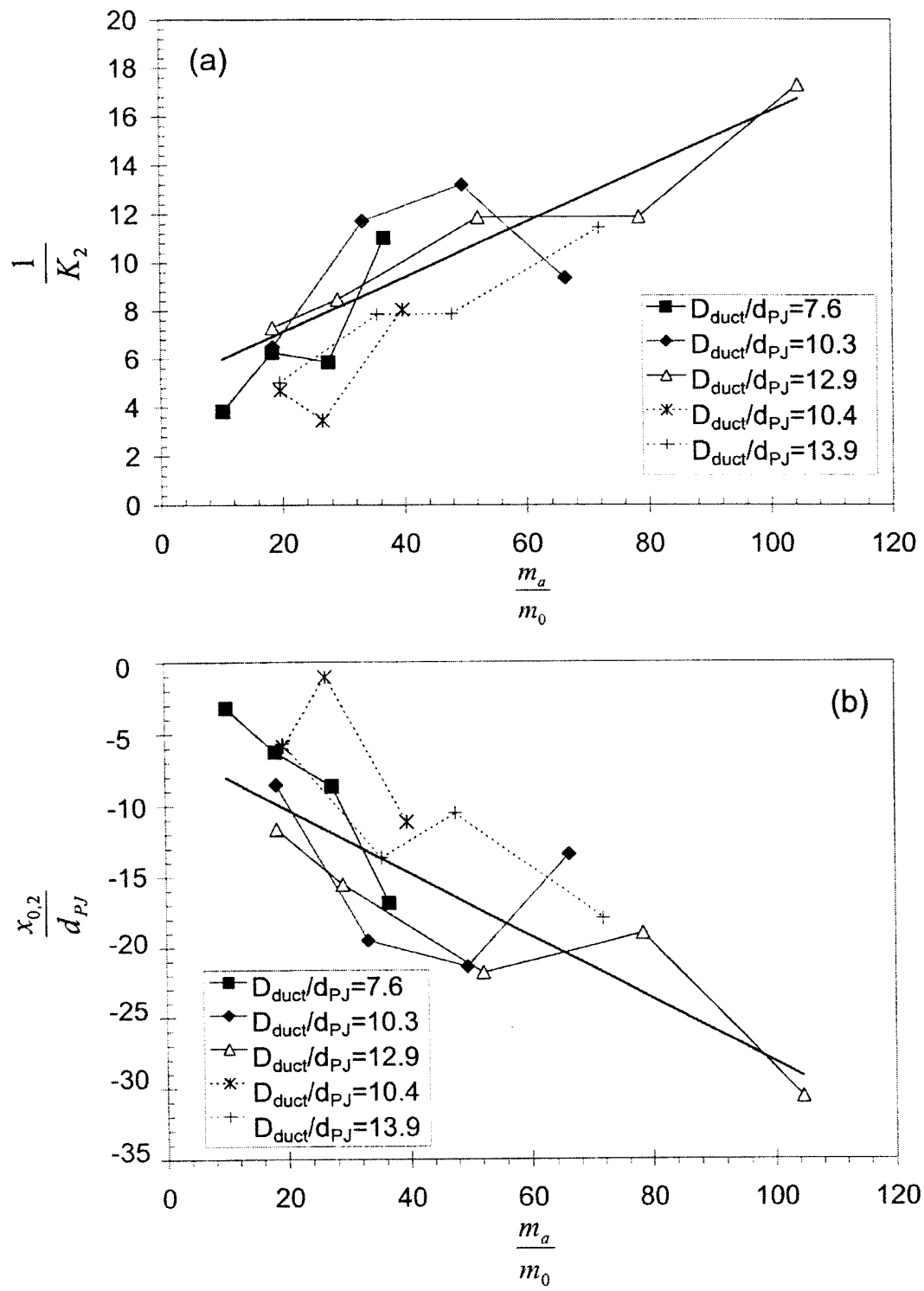

Figure 8. The effect of the mass flux ratio of coflowing fluid to jet fluid on (a) the reciprocal of the far field jet spreading rate, $K_{2}$; and $(b)$ the far-field jet spreading rate virtual origin, $x_{0,2} / d_{\mathrm{PJ}}$, for different confinements and constant PJ nozzle conditions. Solids lines indicate $d_{\mathrm{PJ}}=38 \mathrm{~mm} \mathrm{PJ}$ nozzle results; dashed lines indicate $d_{\mathrm{PJ}}=28 \mathrm{~mm} \mathrm{PJ}$ nozzle results. 
$R$-squared value, as shown in the relevant figures. Hence, the linear best fit of the dependencies results in the following relationships:

$$
\begin{gathered}
K_{1}=0.00774\left(\frac{D_{\text {duct }}}{d_{\mathrm{PJ}}}\right)+0.0948 \\
\frac{x_{0,1}}{D_{\text {duct }}}=-3.386\left(\frac{U_{\mathrm{a}}}{U_{\mathrm{e}-\mathrm{PJ}}}\right)-0.3903
\end{gathered}
$$

A similar analysis was performed to assess the dominant dependencies of the spreading rate in the developed region as characterized by Eq. (5). The $R$-squared statistical measure was used to quantify that $K_{2}$ and $x_{0.2} / d_{\mathrm{PJ}}$ correlated most strongly with the mass flux ratio of the coflowing fluid to the jet fluid, $m_{\mathrm{a}} / m_{0}$. The dependence of either $K_{2}$ or $x_{0.2} / d_{\mathrm{PJ}}$ on the confinement ratio, coflow velocity ratio, or momentum ratio was weaker, as quantified using the $R$-squared measure. Figure $8 a$ demonstrates that the jet spreading rate is inversely proportional to the mass flux ratio, and is described reasonably well by

$$
\frac{1}{K_{2}}=0.1138\left(\frac{m_{\mathrm{a}}}{m_{0}}\right)+4.8311
$$

Likewise the jet concentration half-width (Figure $8 b$ ) is best described by the following relationship:

$$
\frac{x_{0,2}}{d_{\mathrm{PJ}}}=-0.2240\left(\frac{m_{\mathrm{a}}}{m_{0}}\right)-5.7547
$$

Equations (6)-(9) are valid within the bounds of the conditions investigated: $7.6<D_{\text {duct }} / d_{\mathrm{PJ}}<12.8,0.04<U_{\mathrm{a}} / U_{\mathrm{e}-\mathrm{PJ}}<0.19$, and $12<$ $m_{\mathrm{a}} / m_{0}<104$. These relationships are also consistent with experimental results obtained using the secondary $28-\mathrm{mm}$ PJ nozzle with consequently different coflow ratios and confinements.

\section{DISCUSSION}

The primary purpose in developing Eqs. (6)-(9) is to isolate the dominant effects of coflow and confinement on the concentration field of the PJ flow, rather than to use them directly for the prediction of it. A similar analysis, applied to other types of rapidly spreading jet flows of industrial relevance, will likewise reveal the dominant features and appropriate scaling constants for those particular jet nozzles. Equations (6)-(9) reveal 
that, to a first approximation, the jet half-width contour is controlled by the mass flux ratio, the rate of concentration decay by the confinement ratio, and the location of the virtual origin of the concentration decay by the velocity ratio. Maintaining similarity of these dimensionless groups will not only account for these first-order dependencies; it will also account for any of the secondary interdependencies discussed above.

Mixing similarity between two environments of different density ratio can be achieved by the use of Eqs. (1) and (2) and keeping the mass flux ratio and velocity ratio of coflowing fluid to jet fluid constant. For the present PJ flow, Eq. (7) shows that maintaining the velocity ratio will ensure reasonable similarity in the location of the virtual origin $x_{0.1} / D_{\text {duct }}$. Similarly Eqs. (8) and (9) show that maintaining the same mass flux ratio will ensure reasonable similarity of the jet half-width contour, as characterized by $K_{2}$ and $x_{0,2} / d_{\mathrm{PJ}}$. However, Eq. (6) shows that the change in the confinement ratio required by Eq. (1) implies that the concentration decay rate, $K_{1}$, will be different in the two facilities. If uncorrected, this would result in a difference in their mean scalar fields. Therefore, to achieve reasonable first-order similarity of the mean scalar field, it is necessary to apply a further correction. The remaining dimensionless parameter that can be controlled is the simulated air fuel ratio. This can be distorted in the isothermal model by a factor, denoted $\kappa$, which is given by

$$
\kappa=\frac{K_{1, \text { model }}}{K_{1, \text { plant }}}
$$

where $K_{1, \text { model }}$ and $K_{1, \text { plant }}$ are calculated from the confinement ratio using Eq. (6). A distortion of the stoichiometry can be achieved in acid-alkali simulations by changing the concentrations of acid and alkali, and in PLIF experiments by changing the reference concentration used to normalize the raw measurements.

Unfortunately, quantitative assessment of the proposed scaling procedure is extremely difficult because operating rotary kilns are never truly at steady state and their harsh environment makes detailed measurement very difficult. However, some qualitative support for the proposed criterion has been obtained by comparison with the pilot-scale measurements of PJ and momentum dominated flames conducted by Parham et al. (2000a). Kiu et al. (2002) conducted small-scale acid-alkali simulations of the flames in the pilot-scale facility, taking care to simulate the inlet conditions, which were not uniform. They concluded that the modified Thring-Newby criterion proposed here gave better 
qualitative agreement of the flame shape than did the Thring-Newby, Craya-Curtet, or geometric scaling criteria. In addition, Parham (2000) used profiles of mean $\mathrm{CO}$ concentration at a radial traverse in the pilot-scale facility as an indicator of the radial extent of the flame. These were found to compare reasonably well these with the equivalent profiles of jet concentration measured in the present quantitative isothermal PLIF experiments. Although these comparisons are too qualitative to constitute proof, they do provide sufficient support to demonstrate the usefulness of the proposed approach.

\section{CONCLUSIONS}

A PLIF technique for the quantitative measurement of scalar concentration in confined jet flows has been developed and assessed. The scalar mixing field of a nonreacting PJ flow has been quantified for a range of confinements and coflow velocity ratios with a view to providing physical insight into the dominant mixing mechanisms and developing a new criterion for scaling these measurements to large-scale combustion applications. While the experiments, analysis, and results are specific to the processing jet nozzle, the general procedure is also relevant to other complex, unsteady, and rapidly spreading high-Reynolds-number nozzle designs that are used in industrial combustion systems. The scalar measurements also provide new data for the validation of future computational mixing models of this type of jet flow.

The instantaneous PJ flow is dramatically different from the mean flow, being dominated by highly complex and unsteady motions. These features are reproduced in physical models and so highlight the relevance of physical modeling for these flows. The mean flow is characterized by a near-field region of rapid spread, a transition to a region in which the mean flow displays reasonable similarity, termed the "developed" region, and a region of impingement. An assessment is made of the first-order effects of the coflow and confinement based on the characteristics of the developed region using the well-known equations for a free jet. This assessment reveals that, to a first order,

- the centerline scalar decay rate, $K_{1}$, depends on the confinement ratio;

- the location of the centerline concentration decay virtual origin, $x_{0,1}$, depends on the coflow velocity ratio; and

- both the half-width spreading rate, $K_{2}$, and virtual origin, $x_{0,2}$, depend on the mass flux ratio. 
In addition to these dominant relationships, a secondary interdependence of the variables is observed which is deduced to be controlled by the same dimensionless parameters revealed in the first analysis. These dimensionless groups are therefore used to derive a scaling methodology that is appropriate for physically modeling of such rapidly spreading jets.

The analysis of scaling laws found that, to maintain similarity between two systems of different density ratios, it is first necessary to distort the confinement ratio according to a modified form of the ThringNewby parameter. For rapidly spreading jets however, the effect of such a distortion must be further corrected. A distortion of the stoichiometry in the isothermal model, given by the ratio of $K_{1}$ in the model to that in the plant [Eq. (10)] is proposed to achieve this. For the precessing jet nozzle, $K_{1}$ can be related empirically to the confinement ratio [Eq. (6)]. However, the same approach can also be applied to other rapidly spreading jet flows using appropriate empirical data. The limited qualitative comparisons that are available support the proposed scaling procedure, but more quantitative data in large-scale facilities is required for full validation.

\section{REFERENCES}

Becker, H.A., Hottel, H.C., and Williams, G.C. (1963) Mixing and flow in ducted turbulent jets. Proc. Combust. Instit., 9, 7-19.

Becker, H.A., Hottel, H.C., and Williams, G.C. (1967) The nozzle-fluid concentration field of the round, turbulent, free jet. J. Fluid Mech., 30, 2, 285-303. Curtet, R. (1958) Confined jets and recirculation with cold air. Combust. Flame, 2, 383-411.

Dahm, W.J.A. and Dimotakis, P.E. (1990) Mixing at large Schmidt number in the self-similar far field of turbulent jets. J. Fluid Mech., 217, 299-330.

Ellis, J. (2000) An Initial View on Methodologies for Emission Baselines: Cement Case Study. OECD Information Paper, Annex I Expert Group, Paris, http://www.oecd.org/dataoecd/17/12/2390789.pdf (accessed November 2004).

Hill, S.J., Nathan, G.J., and Luxton, R.E. (1992) Precessing and Axial Flows Following a Sudden Expansion in an Axisymmetric Nozzle. Eleventh Australasian Fluid Mechanics Conference, University of Tasmania, Hobart, Australia, 14-18 Dec. 1113-1116.

Jenkins, B.G. (1998) Modeling-It's plastic, mathematic, stochastic, elastic and fantastic. Dev. Chem. Eng. Min. Proc., 7, 3-4.

Karasso, P.S. and Mungal, M.G. (1996) Scalar mixing and reaction in plane liquid shear layers. J. Fluid Mech., 323, 23-63. 
Karasso, P.S. and Mungal, M.G. (1997) Mixing and reaction in curved liquid shear layers. J. Fluid Mech., 334, 381-409.

Kiu, S., Leong, R., and Chen, Y.S. (2002) Validation of Physical and Mathematical Modelling Criteria for Advanced GyroTherm Burners. Internal Report, Department of Mechanical Engineering, University of Adelaide.

Luxton, R.E., Nathan, G.J., and Luminis Pty. Ltd. (1991) Controlling the Motion of a Fluid Jet. U.S. Letters patent 5,060,867.

Manias, C.G. and Nathan, G.J. (1993) The precessing jet gas burner-a low NO burner providing process efficiency and product quality improvements. World Cement, 24, 3, 4-11.

Manias, C.G. and Nathan, G.J. (1994) Low $\mathrm{NO}_{\mathrm{x}}$ clinker production. World Cement, 25, 5, 54-56.

Mi, J. and Nathan, G.J. (2003) The effect of probe resolution on the measurement of a passive scalar and its derivatives. Exp. Fluids, 34, 686-696.

Mi, J., Nobes, D.S., and Nathan, G.J. (2001) Influence of jet exit conditions on the passive scalar field of an axisymmetric free jet. J. Fluid. Mech., 432, 91-125.

Miller, P.L. and Dimotakis, P.E. (1991) Reynolds number dependence of scalar fluctuations in a high Schmidt number turbulent jet. Phys. Fluids A, 3, 5, 1156-1163.

Moles, F.D., Watson, D., and Lain, P.B. (1972) The Aerodynamics of the Rotary Cement Kiln. Fourth Symposium on Flames and Industry, Imperial College, London, pp. 2-10.

Mullinger, P.J. (1999) The relevance of predictive techniques to industrial combustion and heat transfer problems. Dev. Chem. Eng. Min. Proc., 7, 3-4, 225-244.

Nathan, G.J. and Dally, B.B. (2003) Challenges and Progress in the Modeling of Heat Transfer and $\mathrm{NO}_{\mathbf{x}}$ Emissions from Rotary Kiln Flames Involving Unsteady Flows. Third International Conference on CFD in the Minerals and Process Industries, CSIRO, Melbourne, Australia, 10-12 Dec.

Nathan, G.J. and Manias, C.G. (1995) The Role of Process and Flame Interaction in Reducing $\mathrm{NO}_{\mathrm{x}}$ Emissions. Second International Conference on Combustion and Emissions Control, The Institute of Energy, London, pp. 309-318.

Nathan, G.J. Turns, S.R., and Bandaru, R.V. (1996) The influence of jet precession on $\mathrm{NO}_{\mathbf{x}}$ emissions and radiation from turbulent flames. Combust. Sci. Technol., 112, 211-230.

Nathan, G.J., Hill, S.J., and Luxton, R.E. (1998) An axisymmetric 'fluidic' nozzle to generate jet precession. J. Fluid Mech., 380, 347-380.

Newbold, G.J.R. (1997) Mixing and Combustion in Precessing Jet Flows. Ph.D. Thesis, Department of Mechanical Engineering, The University of Adelaide, Australia. 
Newbold, G.J.R., Nathan, G.J., and Luxton, R.E. (1997) Large-scale dynamics of an unconfined precessing jet flame. Combust. Sci. Technol., 126, 1-6, 53.

Newbold, G.J.R., Nathan, G.J., Nobes, D.S., and Turns, S.R. (2000) Measurement and prediction of $\mathrm{NO}_{x}$ emissions from unconfined propane flames from turbulent-jet, bluff-body, swirl and precessing jet burners. Proc. Combust. Instit., 28, 481-487.

Papanicolaou, P.N. and List, E.J. (1988) Investigations of round vertical turbulent buoyant jets. J. Fluid Mech., 195, 341-391.

Parham, J.J. (2000) Control and Optimisation of Mixing and Combustion from a Precessing Jet Nozzle. Ph.D. Thesis, Department of Mechanical Engineering, The University of Adelaide, Australia.

Parham, J.J. Nathan, G.J., Smart, J.P., Hill, S.J., and Jenkins, B.G. (2000a) The relationship between heat flux and $\mathrm{NO}_{\mathrm{x}}$ emissions in gas-fired rotary kilns. J. Inst. Energy, 73, 494, 25-34.

Parham, J.J., Nathan, G.J., and Alwahabi, Z.T. (2000b) The influence of a coflow on the instantaneous structure of a confined precessing jet flow. The Album of Visualization, 17, The Visualization Society of Japan.

Parham, J.J., Nathan, G.J., and Alwahabi, Z.T. (2001) Quantification of Mixing Characteristics for the Optimisation of Combustion in Rotary Kilns. Fourteenth Australasian Fluid Mechanics Conference, University of Adelaide, Adelaide, Australia, 10-14 Dec.

Qamar, N., Nathan, G.J., Alwahabi, Z.T., and King, K.D. (2005) The effect of global mixing on soot volume fraction: Measurements in simple jet, precessing jet and bluff body flames. Proc. Combust. Inst., 30, 1, 1493-1500.

Smith, E., Nathan, G.J., and Dally, B.B. (2003) Assessment of a Simple 2-D Steady State Model of the Flow Field Emerging from the Precessing Jet Nozzle. International Conference on Industrial and Applied Mathematics, Sydney.

Spalding, B. (1962) The art of partial modeling. Proc. Combust. Inst., 9, 833.

Thring, M.W. and Newby, M.P. (1953) Combustion length of enclosed turbulent jet flames. Proc. Combust. Instit., 4, 789-796.

Turns, S.R. and Myhr, F.H. (1991) Oxides of nitrogen emissions from turbulent jet flames: Part I-Fuel effects and flames radiation. Combust. Flame, 87, 319-335.

Videgar, R. (1997) Gyro-Therm technology solves burner problems. World Cement, Nov.

Wong, C.Y., Lanspeary, P.V., Nathan, G.J., Kelso, R.M., and O'Doherty, T. (2003) Phase-averaged velocity in a fludic precessing jet nozzle and in its near external field. J. Exp. Therm. Fluid Sci., 27, 5, 515-524. 